\title{
Prevalencia de infarto criptogénico en pacientes con diagnóstico de infarto cerebral
}

\section{Hernán F. Bayona-Ortiz*, Carlos F. Martínez-Rubio, María C. Valencia-Mendoza, Mariajose Centeno-Padilla y Santiago A. Ortiz-Galindo}

Hospital Universitario Fundación Santa Fe de Bogotá, Departamento de Neurología, Universidad de los Andes, Bogotá, Colombia

Recibido el 12 de mayo de 2016; aceptado el 11 de junio de 2016

Disponible en Internet el 11 de agosto de 2016

\section{PALABRAS CLAVE \\ Ictus; \\ Prevalencia; \\ Enfermedad \\ cerebrovascular; \\ Estudio transversal; \\ Fibrilación auricular}

\begin{abstract}
Resumen
Objetivo: Definir la prevalencia del infarto de origen criptogénico en pacientes con diagnóstico de infarto cerebral isquémico entre los años 2007 a 2014.

Métodos: Se realizó un estudio de tipo corte transversal. Se incluyeron todos los pacientes mayores de 18 años con ataque cerebrovascular isquémico agudo. Se evaluó cada caso mediante una lista de chequeo para la etiología y los estudios de cardiología. Se hizo una descripción de la población y de los factores de riesgo con análisis univariado. La asociación de los criptogénicos y no criptogénicos con las diferentes exposiciones se evaluó con el uso de $\mathrm{Chi}^{2}$ o T de Student de acuerdo con el tipo de variable. Se calcularon las razones de disparidad para los factores de riesgo. Se hizo un análisis multivariado para las variables independientes con $p<0,1$.

Resultados: en 340 pacientes se encontró una prevalencia de evento cerebrovascular criptogénico del 19,71\% ( $n=67)$. Al dicotomizar la edad se observó que la prevalencia de ataque cerebrovascular criptogénico fue mayor en el grupo de $<45$ años $42,31 \%$ vs. $17,83 \% \geq 45$ años $(p=0,003)$, con una razón de prevalencias de 2,37 . Solo la enfermedad coronaria $(p=0,04)$ y la fibrilación auricular $(p<0,0001)$, se relacionaron con los casos no criptogénicos en el modelo multivariado.

Conclusiones: se halló una prevalencia de infarto criptogénico ligeramente inferior a la encontrada en la literatura reciente, que pudiera explicarse por un estudio más profundo en nuestra población o un subregistro de la enfermedad.

(c) 2016 Sociedad Colombiana de Cardiología y Cirugía Cardiovascular. Publicado por Elsevier España, S.L.U. Este es un artículo Open Access bajo la licencia CC BY-NC-ND (http:// creativecommons.org/licenses/by-nc-nd/4.0/).
\end{abstract}

\footnotetext{
* Autor para correspondencia.

Correo electrónico: hernanbayonao@gmail.com (H.F. Bayona-Ortiz).
} 


\section{KEYWORDS}

Stroke;

Prevalence;

Cerebrovascular

disease;

Cross-sectional study;

Atrial fibrillation

\section{Prevalence of cryptogenic stroke in patients with a diagnosis of cerebral infarction}

\begin{abstract}
Objective: To define the prevalence of cryptogenic stroke in patients with a diagnosis of ischemic cerebral infarction between 2007 and 2014.

Methods: A cross-sectional study was performed that included all patients over the age of 18 who had suffered an acute ischemic stroke. Each case was evaluated using a checklist for aetiology and cardiology studies. A description was made of the population and the risk factors with univariate analysis. The association with different exposures of cryptogenic and non-cryptogenic stroke was evaluated using the $\mathrm{Chi}^{2}$ or Student's t-test according to the type of variable. The disparity ratios were calculated for the risk factors. A multivariate analysis was undertaken for the independent variables with $p<0.1$.

Results: a prevalence of $19.71 \%(n=67)$ of cryptogenic stroke was found in 340 patients. When dichotomising age it was observed that the prevalence of cryptogenic stroke was greater in the $<45$ age group, $42.31 \%$ vs $17.83 \%$ in the $\geq 45$ age group $(p=0.003)$, with a prevalence ratio of 2.37. Only coronary heart disease $(p=0.04)$ and atrial fibrillation $(p<0.0001)$, were associated with the non-cryptogenic cases in the multivariate model.

Conclusions: a slightly lower prevalence of cryptogenic stroke was found than that described in the recent literature. This could be explained by a more in-depth study in our population or under-reporting of the disease.

(c) 2016 Sociedad Colombiana de Cardiología y Cirugía Cardiovascular. Published by Elsevier España, S.L.U. This is an open access article under the CC BY-NC-ND license (http:// creativecommons.org/licenses/by-nc-nd/4.0/).
\end{abstract}

\section{Introducción}

De acuerdo con la Asociación Americana del Corazón el infarto cerebral isquémico se define como un episodio de disfunción neurológica focal explicada por un infarto cerebral, espinal o retiniano ${ }^{1}$. Según la clasificación TOAST (Trial of Org 10172 in Acute Stroke Treatment) ${ }^{2}$, la enfermedad cerebrovascular puede dividirse en cinco categorías según la etiología. Uno de los cinco tipos es el infarto criptogénico o indeterminado, el cual se define como infarto cerebral que no es atribuible a una fuente definitiva cardioembólica, de aterosclerosis o de vaso pequeño, a pesar de una extensa evaluación vascular, cardiaca y serológica. Se estima que entre un 10 a $40 \%$ de la enfermedad cerebrovascular es criptogénica ${ }^{3-5}$. En Bogotá se ha descrito previamente que hasta el $32 \%$ de los infartos isquémicos son de origen criptogénico ${ }^{6}$. Sin embargo, por estudios realizados en la población hospitalaria se ha detectado que la tasa de pacientes con diagnóstico de enfermedad criptogénica es menor a la reportada previamente ${ }^{7,8}$.

Es frecuente el infarto cerebral por fibrilación auricular, con un $40 \%$ de casos atribuidos a esta causa. Los pacientes con esta enfermedad tienen infartos más extensos y discapacitantes ${ }^{9}$. Muchos infartos criptogénicos son de fuente embólica. Cuando el origen es incierto se trata de un evento cerebrovasculadr de fuente embólica desconocida ${ }^{10,11}$. La monitorización prolongada de los pacientes con indicio de fuente embólica desconocida, lleva a una mayor detección de aquellos con fibrilación auricular paroxística ${ }^{12-14}$. La aproximación diagnóstica adecuada, puede evidenciar una fibrilación auricular encubierta ${ }^{4,15}$.

El objetivo de este trabajo fue definir la prevalencia de infarto cerebral criptogénico en pacientes con diagnóstico de enfermedad cerebral isquémica entre los años 2007 a 2014.

\section{Materiales y métodos}

Se realizó un estudio de corte transversal para medir la prevalencia de infarto criptogénico en el período 2007-2014, en el que se incluyeron pacientes mayores de 18 años con diagnóstico de enfermedad cerebrovascular isquémica. Se excluyeron pacientes con infarto que hubieran sido estudiados en otra institución antes del ingreso; pacientes con diagnóstico de infarto hemorrágico o de accidente isquémico transitorio; pacientes que no tuvieran estudio imagenológico que confirmara el infarto cerebral junto con un estudio de ecocardiografía al menos, y aquellos quienes pidieron alta voluntaria y fallecieron de manera temprana (menos de 48 horas después el ingreso). Se estudió la totalidad de la población del período por lo tanto no se tomó una muestra.

La información se obtuvo de la historia clínica digital (His Isis), de acuerdo con la base de datos que se lleva prospectivamente en el centro de enfermedad cerebrovascular. Una vez definida la población de estudio, se evaluó cada individuo según 32 variables basadas en la literatura estudiada previamente. Estas variables se categorizaron en seis subgrupos: factores demográficos, morbilidad primaria, comorbilidades, estudios realizados, intervención y diagnósticos etiológicos. Con estos datos se aplicaron listas de chequeo propuestas confirmando que los pacientes con diagnóstico de infarto criptogénico cumplían con todos los parámetros de la definición estipulada por la clasificación TOAST. Adicionalmente, se empleó una encuesta para foramen oval considerado como un potencial factor de confusión. Las encuestas fueron aplicadas por los 
coinvestigadores y rectificadas por uno de los neurólogos del grupo investigador [CM]; las discrepancias encontradas se discutieron por consenso con un tercer autor [HB].

Se describieron las variables del estudio en términos de frecuencia para las variables categóricas, para las continuas se realizaron medidas de tendencia central (media o mediana, desviación estándar y rangos intercuartiles). Para la comparación de las variables categóricas se utilizó la prueba de $\mathrm{Chi}^{2}$ o F de Fischer. En cuanto a las variables continuas se hizo una comparación mediante la T de Student o Mann-Whitney como prueba no paramétrica. Se realizó una dicotomización por la variable edad entre $<45$ años y $\geq 45$ años, con un análisis univariado de las medidas de asociación entre los factores de riesgo para la etiología criptogénica o no criptogénica, y análisis bivariado mediante el cálculo de las razones de disparidad (OR, su sigla en inglés) para infarto criptogénico y cada factor de riesgo. Así mismo, se planeó un análisis multivariado con las variables con valor de $p<0,1$ en el análisis univariado. El valor alfa utilizado fue de 0,05. Se empleó el programa STATA 11.2 para el análisis estadístico.

De acuerdo con la resolución de 8430 de 1983, se trata de una investigación sin riesgo en la que se protegió la identidad de los individuos. Así mismo, fue aprobada por el Comité Corporativo de Ética en investigación de la institución donde se llevó a cabo el estudio.

\section{Resultados}

Un total de 369 pacientes presentaron accidente cerebral isquémico; se excluyeron 29 pacientes por estudios incompletos de ecocardiograma e imágenes cerebrales. Se tomaron 340 pacientes, 165 correspondientes al sexo masculino (48,63\%). La mediana de edad fue de 75 años. En la tabla 1 se describen las variables demográficas y los factores de riesgo. Se encontraron 67 pacientes con infarto criptogénico, para una prevalencia del $19,71 \%$. La etiología más frecuente fue la cardioembólica con $42,94 \%$ en el estudio y en tercer lugar la de vaso grande, con un $18,53 \%$.

La población se dicotomizó de acuerdo con su edad (< 45 años y $\geq 45$ años), en donde se clasificaron los diferentes tipos de evento cerebrovascular (tabla 2). De acuerdo con lo anterior, se descubrió que la prevalencia de infarto criptogénico fue mayor en el grupo de pacientes $<45$ años con $42,31 \%$ con relación al grupo de $\geq 45$ años en donde fue del $17,83 \%$. La razón de prevalencias fue de 2,37 de los pacientes jóvenes $<45$ años comparados con los $\geq 45$ años.

Los estudios realizados a los pacientes fueron principalmente imágenes del cerebro y vasculares, así como de ecocardiografía. En la tabla 3 se describen los principales
Tabla 1 Datos demográficos

\begin{tabular}{ll}
\hline Características & Total $\mathrm{n}=340$ \\
\hline Sexo (femenino) & $175(51,47)$ \\
Edad & $71,51 \pm 15,08$ \\
Peso-kg & $71,77 \pm 14,12$ \\
IMC & \\
$\quad$ Sobrepeso & $42(39,25)$ \\
$\quad$ Obesidad & $15(14,02)$ \\
Escolaridad & \\
$\quad$ Primaria & $10(2,94)$ \\
$\quad$ Secundaria & $18(5,29)$ \\
$\quad$ Profesional & $141(41,47)$ \\
NIHSS & $5\left(2-10^{*}\right)$ \\
Tiempo de ventana & $124(36,58)$ \\
$\quad<4,5 \mathrm{~h}$ & \\
Factores de riesgo & \\
Hipertensión arterial & \\
Diabetes mellitus & $242(71,18)$ \\
Enfermedad coronaria & $66(19,41)$ \\
Antecedente de foramen oval permeable & $73(21,47)$ \\
Fumador & $4(1,18)$ \\
Dislipidemia & $97(28,53)$ \\
Consumo de alcohol & $92(27,06)$ \\
Fibrilación auricular & $36(10,59)$ \\
ACV previo & $93(27,35)$ \\
Familiar con ACV & $68(19,77)$ \\
\hline
\end{tabular}

(\%), p25-p75, \pm desviación estándar, IMC= índice de masa corporal NIHSS = National Institutes of Health Stroke Scale, ACV= ataque cerebro-vascular.

hallazgos encontrados en los estudios de Doppler de vasos de cuello, tomografía axial computarizada, angio-tomografía cerebral/cuello, resonancia cerebral, angio-resonancia y ecocardiograma. Todos los pacientes tuvieron una imagen del cerebro bien sea tomografía cerebral o resonancia cerebral $(n=340)$. A 196 pacientes $(57,65 \%)$ se les practicó tomografía y resonancia magnética, sin diferencias en el número de estudios en los casos criptogénicos de los no criptogénicos $(p=0,13)$. A 295 pacientes $(86,76 \%)$ se les hizo una imagen vascular bien fuera ultrasonido Doppler, angio-tomografía o angiografía por resonancia, sin diferencias entre los infartos criptogénicos de los no criptogénicos $(p=0,65)$. En 174 casos $(51,18 \%)$ se realizaron estudios de imágenes del cerebro sumados a una imagen de los vasos sanguíneos sin encontrar diferencia entre los grupos $(p=0,65)$. Dentro de los estudios de imagen vascular la obstrucción por encima del $50 \%$ se documentó

Tabla 2 Descripción según etiología (TOAST) por edad

\begin{tabular}{lcrr}
\hline & $<45$ años $(\%)$ & $\geq 45$ años $(\%)$ & Total \\
\hline Aterotrombótico & $3(11,54)$ & $60(19,11)$ & $63(18,53)$ \\
Cardioembólico & $5(19,23)$ & $141(44,90)$ & $146(42,94)$ \\
De pequeño vaso (lacunar) & $1(3,85)$ & $42(13,38)$ & $43(12,65)$ \\
De otra causa determinada & $6(23,08)$ & $15(4,78)$ & $21(6,18)$ \\
Criptogénico & $11(42,31)$ & $56(17,83)$ & $67(19,71)$ \\
\hline
\end{tabular}


Tabla 3 Resultados de las imágenes del cerebro

\begin{tabular}{ll}
\hline Tipo de estudio & $\mathrm{n}=340$ \\
\hline Doppler de cuello & $243(71,47)$ \\
Normal & $94(27,65)$ \\
Aumento del espesor íntima-media & $33(9,71)$ \\
Obstrucción $<50 \%$ & $89(26,18)$ \\
Obstrucción $\geq 50 \%$ & $27(7,94)$ \\
Tomografía cerebral & $277(81,47)$ \\
Normal & $126(37,06)$ \\
Signos hiperagudos & $40(11,76)$ \\
Hipodensidad $<1 / 3$ & $85(25,00)$ \\
Hipodensidad $\geq 1 / 3$ & $26(7,65)$ \\
Angio-tomografía cerebral/cuello & $83(24,41)$ \\
Normal & $24(7,06)$ \\
Obstrucción $<50 \%$ & $35(10,29)$ \\
Obstrucción $\geq 50 \%$ & $20(5,88)$ \\
Resonancia cerebral & $259(76,17)$ \\
Normal & $15(4,41)$ \\
Infarto territorio anterior & $162(47,65)$ \\
Infarto territorio posterior & $78(22,94)$ \\
Hemorragia intracerebral & $4(1,18)$ \\
Angio-resonancia cerebral/cuello & $34(10,00)$ \\
Normal & $19(5,59)$ \\
Obstrucción $<50 \%$ & $7(2,06)$ \\
Obstrucción $\geq 50 \%$ & $8(2,35)$ \\
\hline &
\end{tabular}

(\%)

por Doppler (7,94\%), angio-tomografía $(5,88 \%)$ y angioresonancia $(2,35 \%)$. El territorio vascular anterior fue el más comprometido en los estudios de resonancia en el $47,65 \%$ de los casos.

Finalmente, se realizaron estudios de ecocardiografía en la población analizada (tabla 4). De acuerdo con los resultados, se encontró una frecuencia de $6,76 \%$ de pacientes con foramen oval permeable y una de 2,35\% para foramen oval y aneurisma del septo interauricular.

En la tabla 5 se muestra el resultado del análisis univariado y bivariado de acuerdo con presentar o no infarto criptogénico. Entre los factores asociados a infarto criptogénico el más importante fue la edad. En este análisis la hipertensión, la fibrilación auricular, la diabetes mellitus y la enfermedad coronaria se asociaron a eventos no criptogénicos.
Tabla 4 Resultados por ecocardiograma

\begin{tabular}{lc}
\hline Tipo de estudio & $\mathrm{n}=340(\%)$ \\
\hline Ecocardiograma & \\
Transesofágico & $138(40,59)$ \\
Transtorácico & $202(59,41)$ \\
Resultados & \\
Sin foramen oval & $309(90,88)$ \\
Foramen oval permeable & $23(6,76)$ \\
Foramen oval + aneurisma del septo & $8(2,35)$ \\
interauricular & \\
\hline
\end{tabular}

Se hicieron dos modelos para el análisis multivariado; en el primero se incluyó como variable dependiente el infarto criptogénico y como variable independiente la edad, con un OR de 3,37 (IC del 95\% 1,47-7,74; p=0,005). En el segundo modelo se utilizó la misma variable dependiente, adicionándole como independientes hipertensión, enfermedad coronaria, fibrilación auricular y diabetes mellitus (tabla 5). Solo la enfermedad coronaria y la fibrilación auricular se relacionaron con infarto no criptogénico. Al hacer un índice de probabilidad entre ambos modelos, se encontró que el segundo modelo es el que mejor se ajusta ( $\mathrm{LHR}=32,89, \mathrm{p}<0,0001)$, con una discriminación del modelo de 0,75 (análisis ROC) que es adecuado (tabla 6).

\section{Discusión}

Entre la población se halló una prevalencia de ataque cerebrovascular criptogénico del 19,71\%, muy similar a la de un estudio publicado en Chile, que encontró además que la etiología de vaso grande y de cardioembolia fueron las causas más frecuentes, al igual que ocurrió en nuestro caso ${ }^{16}$. El desarrollo tecnológico como la mayor cobertura de población, favorece hallar la etiología de los infartos. Es posible que la capacidad de la institución para realizar un estudio completo explique una menor proporción de casos criptogénicos. El hecho de haber efectuado un estudio retrospectivo en el que la causa no está explícita, hace pensar en un subregistro de infarto criptogénico.

Pese a que el evento cerebrovascular es una patología cuya prevalencia aumenta con la edad, el infarto criptogénico es mucho más frecuente en pacientes jóvenes ${ }^{17}$, lo cual puede explicarse por la menor frecuencia de

Tabla 5 Análisis univariado y bivariado por etiología criptogénica

\begin{tabular}{lcccc}
\hline Factores de riesgo & Criptogénico [+], (\%) & Criptogénico [-], (\%) & Valor de $\mathrm{p}$ & OR (IC 95\%) \\
\hline Edad < 45 & $11(42,31)$ & $56(17,83)$ & 0,0002 & $4,60(1,96-10,70)$ \\
Hipertensión & $39(58,21)$ & $203(74,36)$ & 0,008 & $0,48(0,27-0,88)$ \\
Diabetes & $7(10,45)$ & $59(21,61)$ & 0,03 & $0,42(0,15-0,99)$ \\
Enfermedad coronaria & $7(9,86)$ & $74(24,83)$ & 0,006 & $0,33(0,12-0,77)$ \\
Dislipidemia & $15(22,39)$ & $77(28,21)$ & 0,33 & $0,73(0,36-1,42)$ \\
ACV previo & $15(21,13)$ & $61(20,47)$ & 0,90 & $1,04(0,51-2,02)$ \\
FA & $3(4,48)$ & $90(32,97)$ & $<0,0001$ & $0,62(0,15-1,89)$ \\
Familiar con ACV & $5(7,46)$ & $14(5,13)$ & 0,31 & $1,49(0,40-4,59)$ \\
\hline
\end{tabular}

$\mathrm{FA}=$ fibrilación auricular, $\mathrm{ACV}=$ ataque cerebro-vascular 
Tabla 6 Análisis multivariado (modelo 2)

\begin{tabular}{lcc}
\hline Variable & OR (IC del $95 \%)$ & Valor de $\mathrm{p}$ \\
\hline Edad & $1,91(0,77-4,77)$ & 0,16 \\
Hipertensión & $0,87(0,46-1,65)$ & 0,68 \\
Enfermedad coronaria & $0,38(0,15-0,95)$ & 0,04 \\
Fibrilación auricular & $0,11(0,03-0,38)$ & $<0,0001$ \\
Diabetes mellitus & $0,52(0,21-1,26)$ & 0,15 \\
\hline
\end{tabular}

comorbilidades $^{18}$, en cuyo caso es necesaria una búsqueda más ardua que muchas veces no se completa ${ }^{4,5,17,19}$.

El infarto criptogénico es de suma importancia por las causas subyacentes que tienen posibilidad de tratamiento. La primera está asociada con fenómenos de hipercoagulabilidad junto al foramen oval permeable ${ }^{20}$, la segunda con aterosclerosis aórtica y supraaórtica y la tercera por arritmias ocultas ${ }^{19}$. La fibrilación auricular paroxística con episodios mayores a 30 segundos podría no estar detectándose, dado que muchos eventos son subclínicos ${ }^{20}$. La telemetría cardiaca mayor a 12 horas detecta entre 9 a $18,4 \%$ más episodios de fibrilación en estudios seleccionados, incluido el infarto criptogénico ${ }^{21}$.

No existe aún asociación causal clara de foramen oval permeable con infarto criptogénico dados los resultados negativos del cierre percutáneo de foramen en estudios controlados $^{22-24}$. Llama la atención el bajo reporte de pacientes con foramen oval permeable en nuestro estudio, de acuerdo con lo esperado según las series previas, en las cuales el diagnóstico de esta condición es mucho mayor entre los pacientes con infarto criptogénico que entre la población general ${ }^{19,20}$. Es posible que factores como variabilidad interobservador, limitaciones técnicas o falta de estandarización en la toma del examen, puedan influir en un eventual subregistro. De igual forma, se espera encontrar menos foramen oval permeable en poblaciones con más carga de edad como la de nuestra población.

La mayoría de los casos se estudió con resonancia cerebral, para un total de 259 exámenes. Las imágenes son el primer paso en el diagnóstico de infarto criptogénico. Idealmente, la resonancia descarta la etiología en el caso de infartos lacunares. La sugiere en aquellas imágenes que parecen embólicas. Bien sea con lesiones corticales, con transformación hemorrágica o de eventos en múltiples territorios ${ }^{4,17,19}$. Los estudios de los vasos fueron en su mayoría Doppler de vasos de cuello, angio-tomografía y angio-resonancia. La enfermedad aterosclerótica de vaso grande con obstrucción arterial por encima del $50 \%$ ipsilateral al infarto, descarta la etiología criptogénica ${ }^{19}$.

Al tratarse de un estudio observacional hay sesgos que son inherentes, por ejemplo, el sesgo de información que se relaciona con la calidad de los datos. Por esto se intentó desde el inicio tomar las historias con los datos más completos. No se midió el número de estudios de registro cardíaco de más de 24 horas. Sin embargo, la mayoría de los pacientes en el hospital tiene electrocardiograma y telemetría cardíaca en las primeras 24 horas. Además, dentro de la encuesta se incluyó la presencia de arritmias cardíacas como parte de los estudios. Se halló, así mismo, una pequeña cantidad de personas jóvenes, hecho que limita los resultados respecto a este grupo de edad. Se piensa que hay sub-registro de foramen oval permeable. Si bien se trata de una población en la que se hicieron múltiples pruebas de diagnóstico, que la hacen ver heterogénea en la forma de estudiarse, es muy frecuente en la actualidad que cada caso se deba ver de forma individual. En la institución existe un protocolo de imágenes cerebrales que no ha variado en el periodo del estudio. A los pacientes en fase aguda generalmente se les practica una tomografía del cerebro seguida de una imagen de resonancia. Los estudios angiográficos están sujetos a cada caso en particular.

\section{Conclusiones}

La prevalencia de evento cerebrovascular criptogénico en pacientes con diagnóstico de infarto cerebral isquémico en el período 2007 a 2014, fue menor que la de estudios previos en Colombia. La etiología criptogénica en la actualidad es importante porque es posible que haya una fibrilación auricular que no se haya detectado, sobre todo en los pacientes mayores de 60 años con antecedente de ataque cerebrovascular o en aquellos en quienes se ha diagnosticado infarto criptogénico. Se sospecha de un sub-registro de foramen oval permeable dado el bajo porcentaje encontrado. Nuevos estudios también contribuirán a establecer la relación entre los factores de riesgo y el desarrollo del infarto cerebral y de sus diferentes etiologías.

\section{Responsabilidades éticas}

Protección de personas y animales. Los autores declaran que para esta investigación no se han realizado experimentos en seres humanos ni en animales.

Confidencialidad de los datos. Los autores declaran que han seguido los protocolos de su centro de trabajo sobre la publicación de datos de pacientes.

Derecho a la privacidad y consentimiento informado. Los autores declaran que en este artículo no aparecen datos de pacientes.

\section{Conflicto de intereses}

Los autores declaran no tener conflicto de interés

\section{Agradecimientos}

A Orlando Oliveros, Manuel García, Elisa Sánchez, María Fernanda Torres, Fidel, Juan Camilo Solórzano, María Paula Torres, Esteban Ramírez, Camila Armirola, Stephania Tenorio, por la recolección de la información. A Lina Góez Mogollón por la revisión del análisis y la edición del artículo en su totalidad.

\section{Bibliografía}

1. Sacco RL, Kasner SE, Broderick JP, Caplan LR, Connors JJ, Culebras A, et al. An updated definition of stroke for the $21^{\text {st }}$. century: a statement for healthcare professionals from 
the American Heart Association/American Stroke Association. Stroke. 2013;44(7):2064-89.

2. Adams HJ, Bendixen B, Kappelle L, Biller J, Love B, Gordon D, et al. Classification of subtype of acute ischemic stroke. Definitions for use in a multicenter clinical trial. TOAST. Trial of Org 10172 in Acute Stroke Treatment. Stroke. 1993;24(1):35-41.

3. Kolominsky-Rabas PL, Weber M, Gefeller O, Neundoerfer B, Heuschmann PU. Epidemiology of ischemic stroke subtypes according to TOAST criteria: incidence, recurrence, and longterm survival in ischemic stroke subtypes: a population-based study. Stroke. 2001;32(12):2735-40.

4. Tai WA, Albers GW. Secondary prevention of atherothrombotic or cryptogenic stroke. Circulation. 2014;129(4):527-31.

5. Sacco RL, Ellenberg JH, Mohr JP, Tatemichi TK, Hier DB, Price TR, et al. Infarcts of undetermined cause: the NINCDS Stroke Data Bank. Ann Neurol. 1989;25(4):382-90.

6. Peña I, Ruiz C, Morillo L, Pedraza O, Sanchez E, Sanin L, et al. Acta Neurol Colomb. 2001;17:5.

7. Bayona Ortiz H, Martinez Rubio C, Guio C, Diaz Cruz C. Utilidad del código de ACV en pacientes con accidente cerebrovascular agudo: comparación de dos períodos de tiempo. Acta Neurol Colomb. 2014;30(4):7.

8. Bayona Ortiz H, Diaz Campos A, Jaramillo JA, Posada CL. Concordancia en la realización del triaje entre enfermería y neurología en pacientes con accidente cerebrovascular isquémico en un hospital universitario: un estudio de corte transversal en un hospital de Bogotá. Rev Colomb Enferm. 2015;10:7.

9. Marini C, De Santis F, Sacco S, Russo T, Olivieri L, Totaro R, et al. Contribution of atrial fibrillation to incidence and outcome of ischemic stroke: results from a population-based study. Stroke. 2005;36(6):1115-9.

10. Hart RG, Diener H-C, Coutts SB, Easton JD, Granger CB, O'Donnell MJ, et al. Embolic strokes of undetermined source: the case for a new clinical construct. The Lancet Neurology. 13(4):429-38.

11. Ntaios G, Papavasileiou V, Milionis H, Makaritsis K, Manios E, Spengos K, et al. Embolic strokes of undetermined source in the Athens stroke registry: a descriptive analysis. Stroke. 2015;46(1):176-81.

12. Sanna T, Diener HC, Passman RS, Di Lazzaro V, Bernstein RA, Morillo CA, et al. Cryptogenic stroke and underlying atrial fibrillation. N Engl J Med. 2014;370(26):2478-86.
13. Gladstone DJ, Spring M, Dorian P, Panzov V, Thorpe KE, Hall J, et al. Atrial fibrillation in patients with cryptogenic stroke. $\mathrm{N}$ Engl J Med. 2014;370(26):2467-77.

14. Healey JS, Connolly SJ, Gold MR, Israel CW, Van Gelder IC, Capucci A, et al. Subclinical atrial fibrillation and the risk of stroke. N Eng J Med. 2012;366(2):120-9.

15. Macle L, Cairns JA, Andrade JG, Mitchell LB, Nattel S, Verma A, et al. The 2014 Atrial Fibrillation Guidelines Companion: A Practical Approach to the Use of the Canadian Cardiovascular Society Guidelines. Can J Cardiol. 2015;31(10): 1207-18.

16. Vallejos J, Jaramillo A, Reyes A, Illanes S, Orellana P, Manterola J, et al. Prognosis of cryptogenic ischemic stroke: a prospective single-center study in Chile. J Stroke Cardiovasc Dis. 2012;21(8):621-8.

17. Arauz A, Murillo L, Marquez JM, Tamayo A, Cantu C, Roldan FJ, et al. Long-term risk of recurrent stroke in young cryptogenic stroke patients with and without patent foramen ovale. Int J Stroke. 2012;7(8):631-4.

18. Lamy C, Giannesini C, Zuber M, Arquizan C, Meder JF, Trystram D, et al. Clinical and imaging findings in cryptogenic stroke patients with and without patent foramen ovale: The PFO-ASA Study. Stroke. 2002;33(3):706-11.

19. Liberman AL, Prabhakaran S. Cryptogenic stroke: how to define it? How to treat it? Curr Cardiol Rep. 2013;15(12):423.

20. Saver JL. Cryptogenic stroke. N Eng J Med. 2016;374(21): 2065-74.

21. Kishore A, Vail A, Majid A, Dawson J, Lees KR, Tyrrell PJ, et al. Detection of atrial fibrillation after ischemic stroke or transient ischemic attack: a systematic review and meta-analysis. Stroke. 2014;45(2):520-6.

22. Carroll JD, Saver JL, Thaler DE, Smalling RW, Berry S, MacDonald LA, et al. Closure of patent foramen ovale versus medical therapy after cryptogenic stroke. N Engl J Med. 2013;368(12):1092-100.

23. Furlan AJ, Reisman M, Massaro J, Mauri L, Adams H, Albers GW, et al. Closure or medical therapy for cryptogenic stroke with patent foramen ovale. N Engl J Med. 2012;366(11): 991-9.

24. Meier B, Kalesan B, Mattle HP, Khattab AA, Hildick-Smith $D$, Dudek $D$, et al. Percutaneous closure of patent foramen ovale in cryptogenic embolism. N Engl J Med. 2013;368(12): 1083-91. 